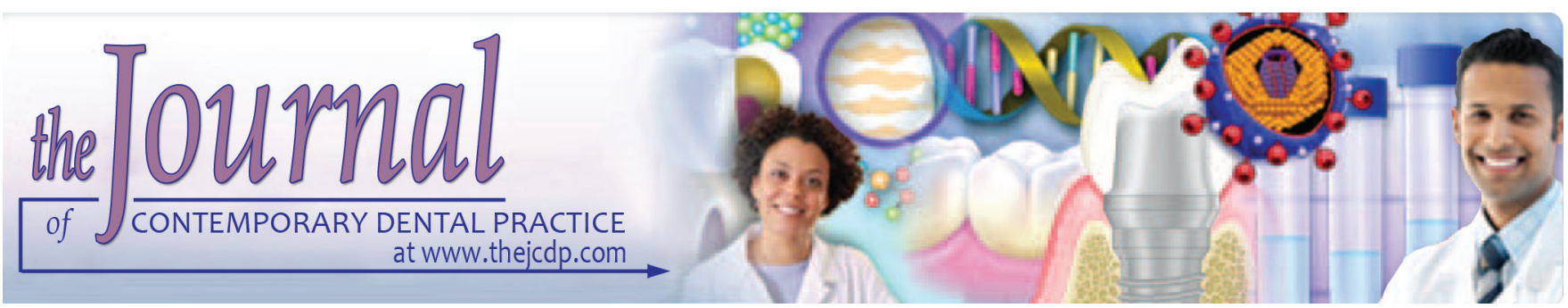

\title{
Retrospective Assessment of Healing Outcome of Endodontic Treatment for Mandibular Molars with C-shaped Root Canal
}

${ }^{1}$ Anika Bansal, ${ }^{2}$ Anuj Singh Parihar, ${ }^{3}$ Achla Sethi, ${ }^{4}$ Kishore Kumar Majety, ${ }^{5}$ Jaina Panjabi, ${ }^{6}$ Basanta Kumar Choudhury

\begin{abstract}
Introduction: A thorough knowledge of the anatomic morphology of the root canal system is necessary for the long-term success of the root canal therapy. The occurrence of $\mathrm{C}$-shaped root canal configuration is one such variation. Achievement of favorable prognosis after commencing root canal therapy in such teeth is one of the challenges imposed on the endodontist. Hence, we evaluated the healing occurring after endodontic therapy in patients with $\mathrm{C}$-shaped root canals in mandibular molars.
\end{abstract}

Materials and methods: The present study was conducted in the Department of Conservative Dentistry of the institution and included assessment of all the patients who underwent root canal treatment of the mandibular first and second molars. Endodontic therapy was performed in all the cases by experienced endodontist. Final postoperative radiographs were taken. Recording of the data of the personal and clinical details of a total of 250 patients was done. All the clinical and radiographic details of the patients, such as tooth location in the jaw, presence or absence of C-shaped canals, status of the pulp tissue, presence or absence of the fractures, and other details of the patients were recorded. Radiographic and clinical examination of the tooth of the patients was done during the baseline visit and further during the follow-up visits. The presence of $\mathrm{C}$-shaped root

\footnotetext{
${ }^{1}$ Department of Conservative Dentistry and Endodontics, Sudha Rastogi College of Dental Sciences and Research, Faridabad Haryana, India

${ }^{2}$ Department of Periodontology, RKDF Dental College \& Research Centre, Bhopal, Madhya Pradesh, India

${ }^{3}$ Conservative Dentistry and Endodontics, Swami Devi Dyal Hospital and Dental College, Panchkula, Haryana, India

${ }^{4}$ Department of Conservative Dentistry and Endodontics, GSL Dental College and Hospital, Rajahmundry, Andhra Pradesh, India

${ }^{5}$ Vancouver Coastal Health, Canada

${ }^{6}$ Department of Oral Medicine \& Radiology, Institute of Dental Science \& Sum Hospital, Bhubaneswar, Orrisa, India

Corresponding Author: Anika Bansal, Department of Conservative Dentistry and Endodontics, Sudha Rastogi College of Dental Sciences and Research, Faridabad, Haryana, India Phone: +919501544877, e-mail: bansalanika9@gmail.com
}

canals was confirmed using the radiographs. Periapical index (PI) scoring system was used. Categories defined for enlisting the healing after the root canal treatment with the assessment of the PI score. All the results were analyzed by Statistical Package for the Social Sciences software.

Results: Vital pulp tissue was encountered in majority of the cases. C-shaped root canal configuration was observed in $40 \%$ of the cases included in the present study. After completion of the endodontic therapy, complete crown placement was done in only $22 \%$ of the cases. In cases of vital teeth with C-shaped root canals configuration, most of the teeth showed complete healing. Significant results were obtained while comparing the complete coverage crown parameter in relation to the healing process in teeth with $\mathrm{C}$-shaped root canals.

Conclusion: In the present study, no significant effect of the $\mathrm{C}$-shaped root canal configuration was found on the healing rate of the endodontic therapy in mandibular molars.

Clinical significance: Meticulous endodontic therapy with special techniques should be done while preparing teeth with C-shaped root canals.

Keywords: C-shaped, Molars, Root canal.

How to cite this article: Bansal A, PariharAS, Sethi A, Majety KK, Panjabi J, Choudhary BK. Retrospective Assessment of Healing Outcome of Endodontic Treatment for Mandibular Molars with C-shaped Root Canal. J Contemp Dent Pract 2017;18(7):591-595.

Source of support: Nil

Conflict of interest: None

\section{INTRODUCTION}

For the long-term success of the root canal therapy, a thorough knowledge of the anatomic morphology of the root canal system is necessary. ${ }^{1}$ Lots of variations occur in the root canal system of the posterior teeth. The occurrence of C-shaped root canal configuration is one such variation. The racial predilection of this variation has also been proved in numerous studies. ${ }^{2}$ Mandibular and maxillary molars are the teeth most commonly affected but this anatomic variation in the root form. One of the challenges 
imposed on the endodontist is the achievement of favorable prognosis after commencing root canal therapy in such teeth. ${ }^{3,4}$ Hence, we evaluated the healing occurring after endodontic therapy in patients with $\mathrm{C}$-shaped root canals in mandibular molars.

\section{MATERIALS AND METHODS}

The present study was conducted in the Department of Conservative Dentistry of the institution which included assessment of all the patients who underwent root canal treatment of the mandibular first and second molars from June 2010 to May 2015. The ethical clearance was obtained from the Institutional Ethical Committee, and written consent was obtained after explaining in details the entire research protocol. Patients who underwent retreatment of the previous failed endodontic therapy or periapical surgery in relation to the mandibular molars were excluded from the present study. Patients with any systemic illness, any known drug allergy, any immunocompromised state or patients in whom follow-up data for a minimum of 1 year was not present were excluded from the present study. Patients were prepared for the endodontic therapy. Rubber isolation was done followed by access preparation using diamond burs. Root ZX was used for the measurement of the working length. Nickeltitanium files were used for the preparation of the apical third of the tooth. For the purpose of irrigation during canal preparation, $2.5 \%$ of sodium hypochlorite was used as an irrigant. Gutta-percha cones and sealer were used for final filling of the canals. Final postoperative radiographs were taken. Recording of the data of the personal and clinical details of a total of 250 patients was done. All the clinical and radiographic details of the patients, such as tooth location in the jaw, presence or absence of $C$-shaped canals, status of the pulp tissue, presence or absence of the fractures, and other details of the patients were recorded.

\section{Evaluation of the Healing}

Radiographic and clinical examination of the tooth of the patients was done during the baseline visit and further during the follow-up visits. The presence or absence of pain as postoperative features was also recorded along with its intensity and type. Both the preoperative and postoperative radiographs were analyzed. The tooth in which more than one root was treated during treatment, the while tooth was considered as a single unit with the minimal value out of the values recorded for all the teeth were taken as standard for comparison.

The presence of C-shaped root canals was confirmed using the radiographs.

- Method previously described in the literature was used for the identification of the C-shaped root canals in the radiographs ${ }^{5}$
- Those teeth in which conical or square root existed and in whom the canals merged into a single major canal were categorized as having C-shaped canals ${ }^{5}$

- Identification of the C-shaped canals was also confirmed by clinical inspection carried out by the endodontists. Periapical index (PI) scoring system:

- Use of periapical and panoramic radiographs was done for the purpose of radiographic evaluation

- For comparing the difference in the status of the periapical tissues before and after the treatment at the time of last follow-up visit of the patients, the PI system was used

- Periapical radiolucencies were graded and scored based on a 5 -point scale ranging from 1 to 5 as shown in Table 1.6,7

Categories defined for enlisting the healing after the root canal treatment with the assessment of the PI score. In various studies on the outcome of root canal therapies, the clinical symptoms and radiographic findings were used as criteria for assessing the healing process. Dammaschke et $\mathrm{al}^{8}$ criteria were used for this categorization which classified the healing into four phases as shown in Table 2. All the results were analyzed by Statistical Package for the Social Sciences software. Chi-square test and multivariate regression analysis were used for the assessment of level of significance.

\section{RESULTS}

Demographic and clinical details of the patients are highlighted in Graph 1. Out of all the 250 cases, 56\% were males while the remaining were females. Involvement of the mandibular molars of the left side was a more common finding in comparison with the molars of the right side. In majority of the cases, vital pulp tissue was

Table 1: Periapical index scoring system

\begin{tabular}{ll}
\hline Score & Parameter \\
\hline 1 & $\begin{array}{l}\text { Healthy periapical part radiographically } \\
2\end{array}$ \\
3 & $\begin{array}{l}\text { Slight alteration in the periapical bone structure } \\
\text { Some amount of mineral loss accompanying the } \\
\text { periapical bone structure loss }(0.5-1 \mathrm{~mm})\end{array}$ \\
4 & $\begin{array}{l}\text { Well-defined radiolucent area accompanying the } \\
\text { demineralization of the periapical bone }(2-4 \mathrm{~mm})\end{array}$ \\
5 & $\begin{array}{l}\text { Exacerbating feature accompanying the } \\
\text { demineralization of the periapical bone }(\geq 5 \mathrm{~mm})\end{array}$ \\
\hline
\end{tabular}

Table 2: Classification used for assessing outcome of healing

\begin{tabular}{ll}
\hline Parameter & Change of PI score \\
\hline Completely healed & 5 to 1,4 to 1,3 to 1,2 to 1,1 to 1 \\
Incompletely healed & 5 to 2,4 to 2,3 to 2,1 to 2,2 to 2 \\
Uncertain healing & 5 to 3,4 to 3,5 to 4,3 to 3 \\
Unsatisfactory & 5 to 5,4 to 5,3 to 5,2 to 5,1 to 5,1 to \\
healing & 3,2 to 3,1 to 4,2 to 4,3 to 4,4 to 4 \\
\hline
\end{tabular}




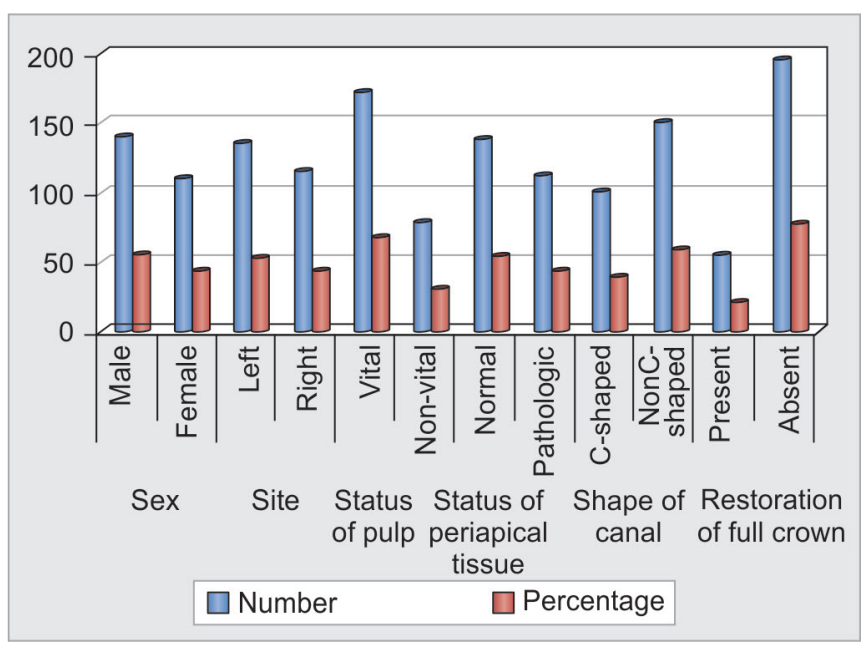

Graph 1: Demographic details of the study

encountered. C-shaped root canal configuration was observed in $40 \%$ of the cases included in the present study. After completion of the endodontic therapy, complete crown placement was done in only $22 \%$ of the cases. Various parameters in relation to the teeth with C-shaped root canal configuration are highlighted in Graph 2. In cases of vital teeth with C-shaped root canal configuration, most of the teeth showed complete healing. In cases with normal periapical tissues, complete healing was achieved in 30 cases, while 13 cases showed unsatisfactory healing. In cases which received full coverage crown, complete healing was observed in six cases. Significant results were obtained while comparing the complete coverage crown parameter in relation to the healing process in teeth with C-shaped root canals.

\section{DISCUSSION}

One of the most important variations occurring in the morphology of root canals is C-shaped configuration. In 1979, Cooke and Cox ${ }^{4}$ were the first to document this variation. Especially among mandibular second molars, the incidence of C-shaped root canals varies from 2.7 to $44.5 \%{ }^{9}$ The main cause for the occurrence of this configuration can be attributed to the Hertwig's root sheath's failure to fuse with the root surfaces. Instead of occurrence of regular pattern of discrete orifices, in cases with C-shaped root canals appearance is of a single ribbon-like orifice. ${ }^{10}$ Ribbon-shaped root canal from the point of orifice to the apex and presence of more than one canal below the C-shaped orifice is the two basic forms of occurrence of the C-shaped root canal. Because of the two-dimensional nature of the radiographs, diagnosis of C-shaped root canals is very difficult solely based on the radiographic techniques. ${ }^{11,12}$ Biomechanical preparation and obturation of the C-shaped root canals are a very tedious and difficult procedure because of its anatomic configuration. Uncertainty also exists in context to continuation of the C-shaped root canal apical third of the root. During the endodontic procedures, there are chances that infected remnants and debris in the root canal with the C-shaped configuration might escape the procedures of irrigation and biomechanical preparation thereby resulting in postoperative pain and treatment failure. Proper obturation techniques are required for completely filling the root canals with such morphological variation to avoid postoperative complications. ${ }^{13-15}$ Hence, we evaluated the healing occurring after endodontic therapy in patients with C-shaped root canals in mandibular molars.

In the present study, we did not observe any significant correlation between the endodontic treatment of the C-shaped root canals and their healing outcomes (Graph 2 and Table 3). Therefore, healing of the root canals is not significantly affected by root shape and morphology. We also observe that in cases without final full-crown restoration, healing decreased, and unsatisfactory healing increased significantly. Our results were in correlation with the results of the previous studies that also noticed complete seal of the root canal sealing

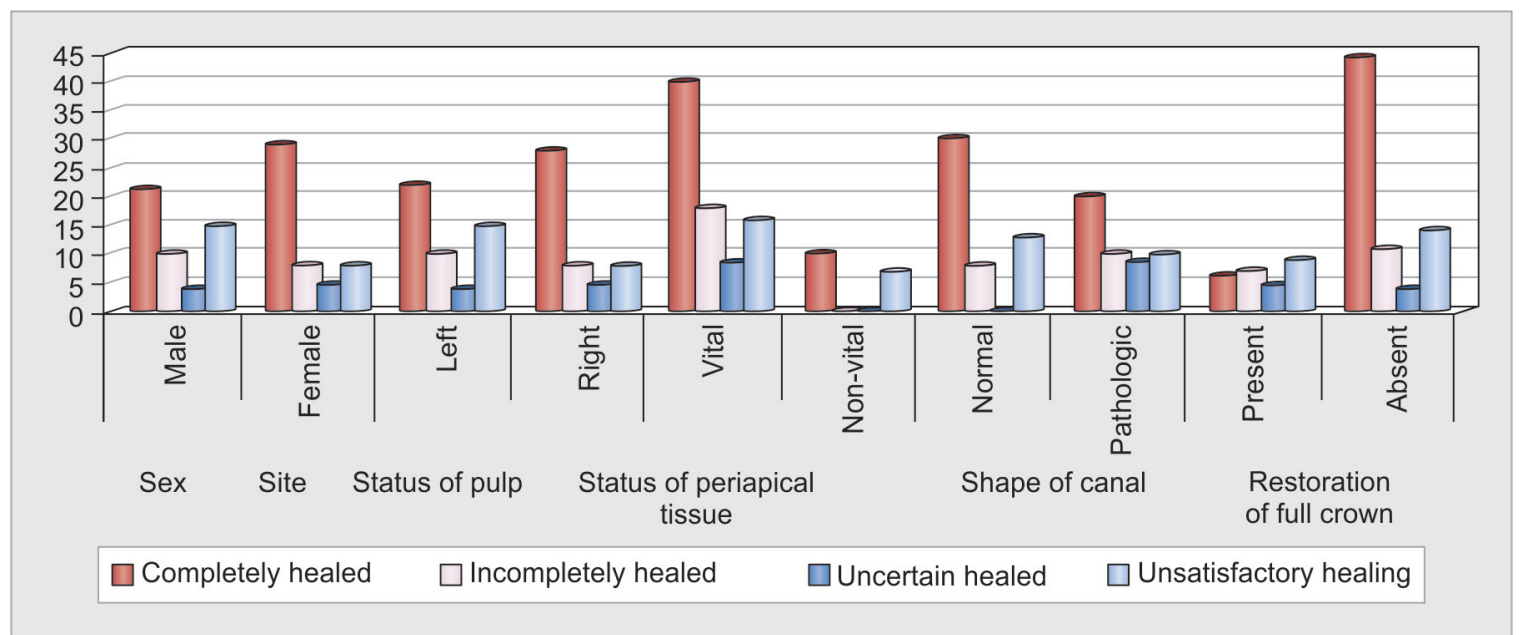

Graph 2: Various parameters in the cases with C-shaped root canals 
Table 3: The p-value for various parameters in cases with C-shaped root canals

\begin{tabular}{|c|c|c|c|c|c|}
\hline Parameter & Completely healed & Incompletely healed & Uncertain healing & Unsatisfactory healing & $p$-value \\
\hline \multicolumn{6}{|l|}{ Sex } \\
\hline Male & 21 & 10 & 4 & 15 & \multirow[t]{2}{*}{0.21} \\
\hline Female & 29 & 8 & 5 & 8 & \\
\hline \multicolumn{6}{|l|}{ Site } \\
\hline Left & 22 & 10 & 4 & 15 & \multirow[t]{2}{*}{0.52} \\
\hline Right & 28 & 8 & 5 & 8 & \\
\hline \multicolumn{6}{|l|}{ Status of pulp } \\
\hline Vital & 40 & 18 & 9 & 16 & \multirow[t]{2}{*}{0.33} \\
\hline Nonvital & 10 & 0 & 0 & 7 & \\
\hline \multicolumn{6}{|c|}{ Status of periapical tissue } \\
\hline Normal & 30 & 8 & 0 & 13 & \multirow[t]{2}{*}{0.17} \\
\hline Pathologic & 20 & 10 & 9 & 10 & \\
\hline \multicolumn{6}{|c|}{ Restoration of full crown } \\
\hline Present & 6 & 7 & 5 & 9 & \multirow[t]{2}{*}{$0.01^{*}$} \\
\hline Absent & 44 & 11 & 4 & 14 & \\
\hline
\end{tabular}

*Significant

materials with coronal protection significantly affects the healing outcome. ${ }^{16,17}$ Jo et al ${ }^{18}$ assessed the frequency of root fusion and $\mathrm{C}$-shaped root canals in maxillary molars and classified the various types of $\mathrm{C}$-shaped root canals by assessment of cone beam computed tomography $(\mathrm{CBCT})$ in a Korean population. They obtained digitalized CBCT images of over 900 subjects from the dental hospital of patients who underwent orthodontic treatment. Retrospective analysis of over 3,500 maxillary molars was done. PiViewstar and Rapidia MPR software displayed the tomographic sections in the axial, coronal, and sagittal planes. In more than $3 \%$ of the cases of the first molars, root fusion was present while in second molars, root fusion was seen in almost $20 \%$ of the cases with a predominance of fusion between mesiobuccal and palatal root. In $<1 \%$ of the cases of first molar, C-shaped root canals were present. They observed a significantly higher frequency of occurrence of root fusion and C-shaped canals in the maxillary second molars in comparison with first molars.

Kim et $\mathrm{al}^{19}$ identified and analyzed the morphology of the root and root canals of the maxillary molars in Korean population with the help of CBCT. They assessed maxillary first and second molars in over 400 patients with CBCT imaging and evaluated their root form and number with Vertucci's classification. They observed that in $0.25 \%$ of the first molar cases, the single root was present. However, in second molar cases, they observed in single root in $4.6 \%$ of the cases. They observed additional canals in more than $60 \%$ of the cases of maxillary first molars. From the results, they concluded that understanding of the root canal anatomy is enhanced by CBCT imaging. Zhang et $\mathrm{al}^{20}$ evaluated the root canal configuration in mandibular molars with $\mathrm{CBCT}$ in Chinese population. They analyzed a total of 389 cases mandibular first and second molars and analyzed their root canal form with the help of CBCT imaging. They observed that two separate roots of mandibular molars were observed in majority of the cases. In first molars cases, three-rooted shapes were observed in $29 \%$ of the cases. From the results, they concluded that among Chinese population, three-rooted mandibular first molars and C-shaped second molars had the highest incidence in their study.

Ahn et $\mathrm{al}^{21}$ assessed the healing rate in C-shaped mandibular molars in patients undergoing nonsurgical endodontic therapy. They analyzed the clinical and radiographic changes occurring in patient who underwent endodontic therapy. For comparing the healing outcome, they used the PI scoring system. They also evaluated the demographic details along with clinical data. They observed that in mandibular molars with C-shaped root canals, total healing rate was approximately $70 \%$. From the results, they concluded that C-shaped morphology of root canals had no effect on the healing phase of mandibular molars undergoing root canal therapy. Silva et al ${ }^{22}$ evaluated the morphology of root canal of the maxillary molars in Brazilian population with the help of CBCT. They assessed a total of 620 patients, in which they analyzed 314 maxillary first molars and 306 maxillary second molars. They evaluated all the variations in the root form of the teeth. A higher prevalent rate of three roots in the molars was observed. In more than $42 \%$ of the cases, mesiobuccal root presented with two root canals. More anatomic variation was seen in the maxillary second molars. From the results, they concluded that in the endodontic diagnosis and treatment planning, $\mathrm{CBCT}$ plays an important role.

\section{CONCLUSION}

No significant effect of C-shape anatomic configuration of the root canals was found on the healing rate of the mandibular molars in the present study. Complete sealing 
of the crown portion of the tooth after root canal therapy completion was the only factor that was found to have a significant effect on the healing rate of mandibular molars with C-shaped root canal configuration.

\section{REFERENCES}

1. Manning SA. Root canal anatomy of mandibular second molars. Part I. Int Endod J 1990 Jan;23(1):34-39.

2. Melton DC, Krell KV, Fuller MW. Anatomical and histological features of C-shaped canals in mandibular second molars. J Endod 1991 Aug;17(8):384-388.

3. Chai WL, Thong YL. Cross-sectional morphology and minimum canal wall widths in C-shaped roots of mandibular molars. J Endod 2004 Jul;30(7):509-512.

4. Cooke HG 3rd, Cox FL. C-shaped canal configurations in mandibular molars. J Am Dent Assoc 1979 Nov;99(5):836-839.

5. Fan B, Cheung GS, Fan M, Gutmann JL, Fan W. C-shaped canal system in mandibular second molars: part II - radiographic features. J Endod 2004 Dec;30(12):904-908.

6. Doyle SL, Hodges JS, Pesun IJ, Law AS, Bowles WR. Retrospective cross sectional comparison of initial nonsurgical endodontic treatment and single-tooth implants. J Endod 2006 Sep;32(9):822-827.

7. Tolias D, Koletsi K, Mamai-Homata E, Margaritis V, Kontakiotis E. Apical periodontitis in association with the quality of root fillings and coronal restorations: a 14-year investigation in young Greek adults. Oral Health Prev Dent 2012 Jun;10(3):297-303.

8. Dammaschke T, Steven D, Kaup M, Ott KH. Long-term survival of root-canal-treated teeth: a retrospective study over 10 years. J Endod 2003 Oct;29(10):638-643.

9. Jafarzadeh $\mathrm{H}, \mathrm{Wu} \mathrm{YN}$. The C-shaped root canal configuration: a review. J Endod 2007 May;33(5):517-523.

10. Fan B, Min Y, Lu G, Yang J, Cheung GS, Gutmann JL. Negotiation of C-shaped canal systems in mandibular second molars. J Endod 2009 Jul;35(7):1003-1008.

11. ManningSA. Root canal anatomy of mandibular second molars. Part II. C-shaped canals. Int Endod J 1990 Jan;23(1):40-45.
12. Sabala CL, Benenati FW, Neas BR. Bilateral root or root canal aberrations in a dental school patient population. J Endod 1994 Jan;20(1):38-42.

13. Velmurugan N, Sandhya R. Root canal morphology of mandibular first premolars in an Indian population: a laboratory study. Int Endod J 2009 Jan;42(1):54-58.

14. Khedmat S, Assadian H, Saravani AA. Root canal morphology of the mandibular first premolars in an Iranian population using cross-sections and radiography. J Endod 2010 Feb;36(2):214-217.

15. Sandhya R, Velmurugan N, Kandaswamy D. Assessment of root canal morphology of mandibular first premolars in the Indian population using spiral computed tomography: an in vitro study. Indian J Dent Res 2010 Apr-Jun;21(2):169-173.

16. Ray HA, Trope M. Periapical status of endodontically treated teeth in relation to the technical quality of the root filling and the coronal restoration. Int Endod J 1995 Jan;28(1):12-18.

17. Katebzadeh N, Sigurdsson A, Trope M. Radiographic evaluation of periapical healing after obturation of infected root canals: an in vivo study. Int Endod J 2000 Jan;33(1):60-66.

18. Jo HH, Min JB, Hwang HK. Analysis of C-shaped root canal configuration in maxillary molars in a Korean population using cone-beam computed tomography. Restor Dent Endod 2016 Feb;41(1):55-62.

19. Kim Y, Lee SJ, Woo J. Morphology of maxillary first and second molars analyzed by cone-beam computed tomography in a Korean population: variations in the number of roots and canals and the incidence of fusion. J Endod 2012 Aug;38(8):1063-1068.

20. Zhang R, Wang H, Tian YY, Yu X, Hu T, Dummer PM. Use of cone-beam computed tomography to evaluate root and canal morphology of mandibular molars in Chinese individuals. Int Endod J 2011 Nov;44(11):990-999.

21. Ahn HR, Moon YM, Hong SO, Seo MS. Healing outcomes of root canal treatment for C-shaped mandibular second molars: a retrospective analysis. Restor Dent Endod 2016 Nov;41(4):262-270.

22. Silva EJ, Nejaim Y, Silva AI, Haiter-Neto F, Zaia AA, Cohenca N. Evaluation of root canal configuration of maxillary molars in a Brazilian population using cone-beam computed tomographic imaging: an in vivo study. J Endod 2014 Feb;40(2):173-176. 